\title{
Age at infection as a determinant of the behavioral effects of herpes encephalitis in mice
}

\author{
DENNIS J. McFARLAND, EDWARD SIKORA, and JOHN HOTCHIN \\ Division of Laboratories and Research, New York State Department of Health, Albany, New York 12201
}

\begin{abstract}
The influence of age at infection on the nature of the behavioral effects of herpes simplex type 1 encephalitis in mice was examined. Weanling and adult mice were initially immunized with a footpad (FP) inoculation of virus, followed 2 weeks later by an intracerebral (IC) inoculation. Subsequent open-field testing 2 weeks later revealed that weanling mice were hypoactive and showed a reduced proportion of center-field entries. In contrast, adult mice were hyperactive. No significant effects on Y-maze alternation or activity were observed. These results indicate that the qualitative nature of the effects of herpes encephalitis on behavior depends upon characteristics of the host.
\end{abstract}

Herpes simplex virus (HSV) is the major cause of sporadic viral encephalitis in the United States (Merritt, 1973). As noted by Baringer (1974), the clinical course of HSV encephalitis is quite unpredictable. In adults, this condition is generally associated with neurological signs, seizures, and personality changes (Williams $\&$ Lerner, 1978). Memory disturbances represent the most consistent residual effect in such cases (Hierons, Janota, \& Corsellis, 1978). In contrast with the typical clinical picture, however, HSV encephalitis may be present as a psychiatric disturbance in the absence of gross neurological effects. Reports in the literature have associated HSV with catatonic stupor (Raskin \& Frank, 1974), a partial Kluver-Bucy syndrome (Shoji, Teramoto, Satowa, \& Narita, 1979), mania (Koehler \& Guth, 1979), and functional psychosis (Wilson, 1976).

The behavioral effects of experimental HSV infection have not been extensively examined. Lycke, Norby, and Roos (1974) found increased locomotor activity and aggression in mice 4 to 5 days following IC inoculation of HSV. This study involved the acute disease, since convulsions and death followed shortly. In contrast, Seegal and Hotchin (1978) observed decreased spontaneous and dl-amphetamine-induced locomotor activity for up to 1 month following subacute HSV infection. In this study, mice were immunized by FP inoculation prior to IC inoculation, which greatly reduced mortality and allowed for the study of chronic effects. To some extent, these differences in results could reflect variations in the conditions of testing and the types of activity measures employed, since such variables often affect the outcome of activity studies (e.g., Valle, 1970). Alternately, other variables relating to the characteristics of the host and the nature of the infection may be operating here.

A variety of host-related factors have been found to influence characteristics of HSV infection in ex- perimental animals. Hirsch, Zisman, and Allison (1970) reported an age-dependent resistance to low doses of HSV, which developed in the first few weeks of life. Kristensson and Sourander (1969) found that HSV infection in weanling animals resulted in the most marked pathology being located in the hippocampus. In contrast, Kristensson (1976) found HSV in suckling animals to have a predilection for undifferentiated neuroectodermal cells. The specific strain of HSV appears also to be of importance, since Rajcani and Szanto (1973) observed that immunofluorescence appeared first in the meninges, gray matter, or white matter, depending upon which of $10 \mathrm{HSV}$ type 1 strains were employed. Thus, factors such as the age of the host and viral genetics have been shown to influence parameters such as mortality and tissue trophism.

In the present study, the relevance of age at infection to the nature of the behavioral effects of experimental HSV encephalitis was examined. Mice were first immunized by means of a FP inoculation, followed 2 weeks later by an IC inoculation. This procedure provided a preparation in which acute mortality was low, allowing for the study of an attenuated form of encephalitis.

\section{METHOD}

A human isolate of HSV type 1, previously examined in this laboratory (Seegal \& Hotchin, 1978; Seegal, Sikora, \& Hotchin, 1980), was employed. The stock virus suspension had a titer of $9.9 \times 10^{5}$ mouse LD $50 / \mathrm{ml}$ (by IC tradition in Nya:NYLAR mice, combined with Reed-Muench end point assessment). The diluent consisted of $.05 \%$ gelatin in $\mathrm{pH} 6.8$ Tris-buffered Hanks balanced salt solution (GTH).

Weanling (4 weeks old) and adult (8 weeks old) female Nya: NYLAR mice received $.05 \mathrm{ml}$ FP inoculations of either a $10^{-2}$ dilution of stock virus suspension or diluent. Two weeks later, these same animals were given identical IC doses of HSV or diluent.

Prior to IC inoculations, animals were housed in groups of 18 in metal cages. Following infection, all animals were housed in 
plastic suspended cages in groups of four or five. At all times, food and water were available ad lib, and the room was maintained on a 10-h-on/14-h-off lighting schedule.

All testing was conducted during the light phase and was accomplished with the aid of an ACT/INTERACT laboratory computer system. Two weeks following IC infection, 14 subjects from each group were tested, first for open-field activity and then for Y-maze exploration.

The open field was a $51 \times 51 \times 45 \mathrm{~cm}$ plywood box painted flat black. Two banks of four photocells each were located on adjacent walls, $2.5 \mathrm{~cm}$ from the floor, with infrared light sources opposite to each. The system was programmed not to count two or more successive breaks of the same photocell within a bank of four photocells. This procedure greatly reduced the sensitivity of the apparatus to such behaviors as sniffing, rearing, and grooming. The mouse was placed in an enclosed $10.5-\mathrm{cm}$ square formed by two corner walls and a removable L-shaped bracket $45-\mathrm{cm}$ high. After $30 \mathrm{sec}$, this barrier was removed, and the subject's latency to ambulate was recorded, with total activity and centerfield activity being recorded in 5-min blocks during a 20-min session.

Spontaneous alternation was examined in a $27 \times 7.5 \times 10 \mathrm{~cm}$ symmetrical Plexiglas Y-maze painted flat black. Photocells, illuminated by infrared LEDs, were located $10 \mathrm{~cm}$ within each arm and $2.5 \mathrm{~cm}$ from the floor. The system was programmed not to count two or more successive breakings of the same photocell. The mouse was placed at the far end of one arm, and the maze was covered. The total number of arms entered and the number of least recently entered arms (alternations) were recorded during a 20 -min session.

\section{RESULTS}

Two mice in the weanling group died following FP inoculation. There were no deaths in any of the other groups.

Analysis of variance of open-field latency scores indicated that only the effect of age was significant $(p<.05)$. Older animals in both the infected and

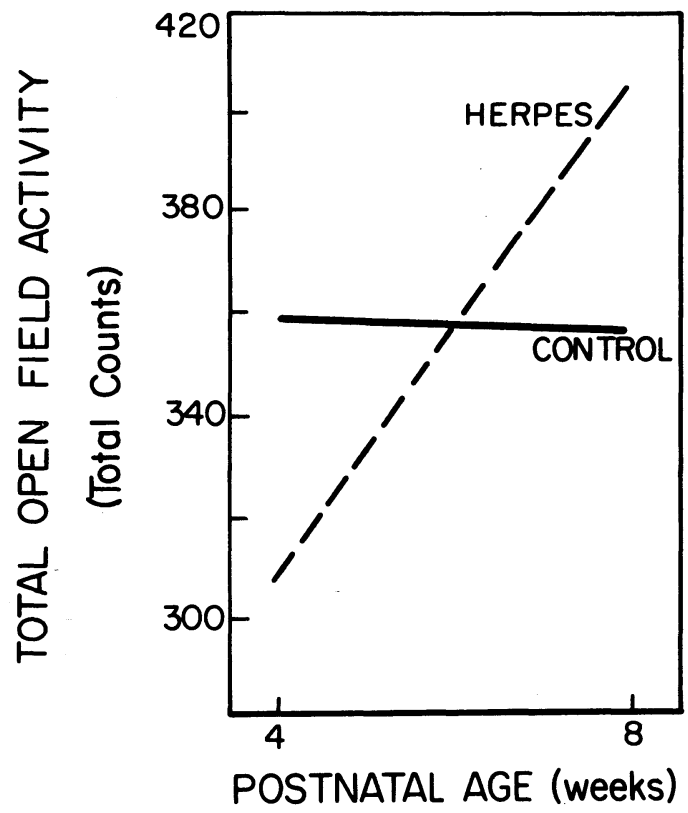

Figure 1. Iotal open-field activity as a function of age at inlection.

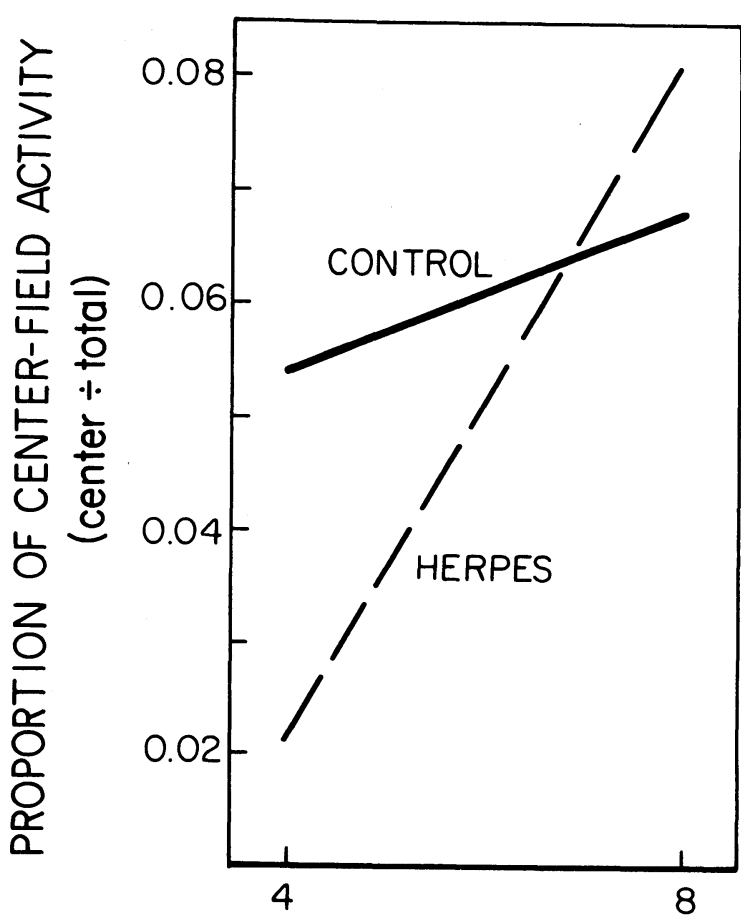

POSTNATAL AGE (weeks)

Figure 2. Proportion of center-field activity as a function of age at infection.

control groups had shorter latencies to ambulate than did weanling animals.

Total open-field activity as a function of virus infection and age is shown in Figure 1. Analysis of variance for this measure resulted in a significant Age by Infection interaction $(p<.05)$. Post hoc analysis (least significant difference test) indicated that weanling herpes-infected animals had lower activity levels than did corresponding controls $(p<.01)$. In contrast, adult HSV-infected animals were significantly more active than were adult controls $(p<.01)$.

The proportion of center-field crosses [center crosses/(center + peripheral crosses)] is shown in Figure 2. Analysis of variance resulted in significant effects for age $(p<.01)$ and the Age by Infection interaction $(p<.01)$. Post hoc analysis indicated that weanling infected animals had a significantly lower proportion of center-field activity when compared with controls $(p<.01)$, while adult infected and control animals were not significantly different. It should be noted that there was a trend toward elevated center-field activity in the adult infected group, however.

There were no significant effects for virus infection or interactions of infection with age for either Y-maze alternation or arms entered. For weanling control and infected animals, the means for percent alternation were $66.5 \%$ and $64.5 \%$, respectively. For adult control and infected animals, the means for percent 
alternation were $66.7 \%$ and $67.3 \%$, respectively. The mean number of arms entered by weanling control animals was 90.3 , while that for infected weanling animals was 86.2. For adult control and infected animals, the means for the number of arms entered were 79.9 and 79.2 , respectively.

\section{DISCUSSION}

The results of the present study demonstrate that the qualitative nature of the behavioral effects of HSV infection in mice depends upon the age at which the animal is infected. It should be noted that the decreased activity level observed in the weanling infected group in this study is consistent with previous reports from this laboratory, in which it was shown that mice infected with the same strain of HSV at essentially the same age were found to be hypoactive (Seegal \& Hotchin, 1978; Seegal et al., 1979). In view of the differences between the results reported in these studies and those of Lycke et al. (1974), the present findings are of interest, since they suggest that there is not an invariant behavioral syndrome associated with herpes encephalitis in mice. However, it is not possible to explain the differences between these studies on the basis of age of infection, since both groups of investigators employed weanling animals. Rather, differences in other parameters, such as the host and viral genotypes and whether or not the animals were immunized prior to IC infection, are probably responsible for these different effects.

The qualitative nature of the behavioral effects of infection with the scrapie virus has been shown to depend upon both the host and viral genotype (McFarland, Baker, \& Hotchin, 1980). The present results demonstrate that the importance of host characteristics as determinants of the behavioral effects of CNS viral infection is not peculiar to the scrapie virus. Indeed, characteristics such as age and genetic background of the host may be important determinants of the specific behavioral syndromes resulting from either clinical or experimental encephalitis produced by many viral agents.

As noted earlier, the effects of HSV encephalitis reported in the clinical literature are quite variable. However, it would appear that the specific symptoms observed do not occur by chance but, rather, are the result of an interaction between characteristics of the host and viral agent.

\section{REFERENCES}

Baringer, J. R. Human herpes simplex virus infections. $A d$ vances in Neurology, 1974, 6, 41-51.

Hierons, R., Janota, I., \& Corsellis, J. A. N. The effects of necrotizing encephalitis of the temporal lobes and limbic areas: A clinico-pathological study of 10 cases. Psychological Medicine, 1978, 8, 21-42.

Hirsch, M. S., Zisman, B., \& Allison, A. C. Macrophages and age-dependent resistance to herpes simplex virus in mice. Journal of Immunology, 1970, 104, 1160-1165.

Koenler, K., \& Guth, W. The mimicking of mania in 'benign' herpes simplex encephalitis. Biological Psychiatry, 1979, 14, 405-411.

KRISTENSSON, K. Experimental herpes simplex virus infection in the immature mouse brain. Acta Neuropathologica, 1976, 35, 343-351.

Kristensson, K., \& Sourander, P. Changes in vascular permeability of the central nervous system by experimental herpes simplex and vaccinia virus infections. Acta Neuropathologica, $1969,14,38-51$.

Lycke, E. R., Norby, B. E., \& Roos, B. Influence of changes in brain monoamine metabolism on behavior of herpes simplex infected mice. Journal of Neurological Sciences, 1974, 22, 277-289.

McFarland, D. J., Baker, F. D., \& Hotchin, J. Host and viral genetic determinants of the behavioral effects of scrapie encephalopathy. Physiology \& Behavior, 1980, 24, 911-914.

Merritt, H. H. A textbook of neurology. Philadelphia: Lea \& Febiger, 1973.

RAJCANI, J., \& Szanto, J. Pathogenicity of laboratory-maintained and low-passaged strains of herpes virus hominis in suckling mice: A comparative fluorescent antibody study. Acta Virologica, 1973, 17, 227-236.

RASkin, D. E., \& Frank, S. W. Herpes encephalitis with catatonic stupor. Archives of General Psychiatry, 1974, 31, 544-546.

Seegal, R., \& Hotchin, J. Effects of herpes virus and amphetamine on locomotor activity. Birth Defects: Original Article Series, 1978, 14, 179-184.

Seegal, R. F., Sikora, E., \& Hotchin, J. Locomotor effects of catecholaminergic drugs on herpes-infected mice. Pharmacology, Biochemistry, and Behavior, 1980, 12, 61-66.

Shoji, H., Teramoto, H., Satowa, H., \& Narita, Y. Partial Kluver-Bucy syndrome following probable herpes simplex encephalitis. Journal of Neurology, 1979, 221, 163-167.

VALLE, F. P. Effects of strain, sex, and illumination on open field behavior of mice. American Journal of Psychology, 1970, 883, 103-111.

Williams, B. B., \& Lerner, A. M. Some previously unrecognized features of herpes simplex encephalitis. Neurology, 1978, 28, 1193-1196.

WiLson, L. G. Viral encephalopathy mimicking functional psychosis. American Journal of Psychiatry, 1976, 133, 165-170.

(Received for publication October 27, 1980; revision accepted January 21, 1981.) 